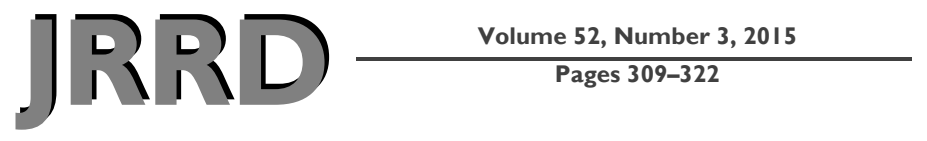

\title{
Efficacy of simple integrated group rehabilitation program for patients with knee osteoarthritis: Single-blind randomized controlled trial
}

\author{
Flávio S. da Silva, MS; ${ }^{1}$ Flávio E. S. de Melo, PT; ${ }^{2}$ Marcelo M. G. do Amaral, PT; ${ }^{2}$ Vinícius V. A. Caldas, PT; ${ }^{2}$ \\ Íria Lúcia D. Pinheiro, PT; ${ }^{2}$ Bento J. Abreu, PhD; ${ }^{*}$ Wouber H. Brito Vieira, PhD ${ }^{2}$ \\ ${ }^{1}$ Department of Morphology, Biosciences Center, and ${ }^{2}$ Department of Physiotherapy, Health Sciences Center, Federal \\ University of Rio Grande do Norte, Lagoa Nova, Natal, Brazil
}

\begin{abstract}
We investigated the role of an evidence-based integrated group rehabilitation program on the treatment of patients with knee osteoarthritis (KOA). This was a two-group, randomized controlled, 8 wk trial with 41 patients with moderate to very severe KOA. Patients were assigned to an intervention group (IG) or control group (CG). After both groups had received a self-management education program, IG participants underwent a rehabilitation program, including educational aspects about KOA followed by exercises. CG participants received only general health orientation about KOA during this period. The outcome measures were the Lequesne algofunctional index; 36-Item Short Form Health Survey (SF-36); and chair-stand, sit-and-reach, timed up-and-go, and 6-minute walk tests. Analysis of covariance revealed significant postintervention improvements of IG participants compared with CG participants $(p<0.05)$ on Lequesne total score and pain and function subdomains; SF-36 physical function, role physical, bodily pain, general health, vitality, and role emotional subdomains; and performance assessed by chair-stand, timed up-and-go, and 6-minute walk tests. Focusing on the primary outcome (Lequesne total score), the mean $+/-$ standard deviation after 8 wk was $5.50+/-2.98$ for the IG and $7.87+/-3.48$ for the CG $(p=0.009)$. The corresponding effect size (partial eta squared with $90 \%$ confidence interval) was $0.23(0.04-0.42)$, indicating a large effect. The presented rehabilitation program reduced pain and improved quality of life and function in patients with KOA.
\end{abstract}

Clinical Trial Registration: ClinicalTrials.gov; Progressive Collective-exercise Program on the Knee Osteoarthritis; NCT01850862; $\quad$ https://clinicaltrials.gov/ct2/show/ NCT01850862?term $=$ NCT01850862\&rank $=1$
Key words: exercise therapy, group, knee, Lequesne, osteoarthritis, physical activity, randomized controlled trial, rehabilitation, self-management, stretching.

\section{INTRODUCTION}

Osteoarthritis is the most common degenerative joint disorder worldwide, especially in the elderly, and a major cause of pain and disability [1]. The most affected joints are the hip and knee, which usually present with loss of articular cartilage, narrowing of joint space, and production of bone spurs [2]. The etiology of knee osteoarthritis (KOA) is multifactorial and includes several conditions such as aging, heredity, obesity, sex, and mechanical overload [3]. Patients with KOA often report muscle weakness, pain, and decreased range of motion. In turn, these symptoms are associated with limited functional

\footnotetext{
Abbreviations: $\mathrm{ANCOVA}=$ analysis of covariance, $\mathrm{CG}=$ control group, $\mathrm{CI}=$ confidence interval, $\mathrm{IG}=$ intervention group, $\mathrm{KOA}=$ knee osteoarthritis, $\mathrm{MCID}=$ minimum clinically important difference, $\mathrm{MDE}=$ minimum detectable effect, SF-36 $=36$-item Short Form Health Survey.

*Address all correspondence to Bento J. Abreu, PhD; Federal University of Rio Grande do Norte-Department of Morphology, Center of Biosciences, BR 101-Lagoa Nova, Natal, Rio Grande do Norte 59067-480, Brazil; +55 843215 3431; fax: +55 843211 9207. Email: abreubj@gmail.com http://dx.doi.org/10.1682/JRRD.2014.08.0199
} 
capacity and decreased quality of life. Thus, the goal of clinical treatment is to improve the patient's health condition, and one major conservative intervention involves physical activity. In fact, regular exercise is associated with significant improvements in pain and disability in patients with symptomatic KOA [4].

In recent work conducted by Holden et al., it was found that older adults were uncertain about the benefits of exercise for knee pain, and therefore, their attitudes and beliefs would affect exercise prescription for these individuals [5]. To be effective, the physical activity program should include patient counseling and education in order to promote positive changes in lifestyle, such as increased daily physical activity and understanding and coping with problems and limitations [6-8]. Moreover, when physical activities are performed in groups, they may reach a larger number of people, improve interpersonal relationships and adherence to guidelines, and provide reduction in health costs [9-10]. Group rehabilitation programs have become more popular in recent years, and a typical program consists of stretching, strengthening resistance, and/or aerobic exercises [1112]. Despite a considerable amount of rehabilitation programs, few works report the efficacy of an integrated group rehabilitation program for subjects with KOA [1314]. Moreover, the exercise protocols are commonly established by the therapist's personal preferences and availability, which hinders the elucidation of which rehabilitation techniques are actually positive for KOA rehabilitation and patient adherence.

Based on the efficacy of various intervention techniques described in previous works $[7,13,15]$, we designed a simple and evidence-based 8 wk group rehabilitation program consisting of exercise and a self-management program for patients with moderate to very severe KOA. Thus, we hypothesized that this integrated group rehabilitation program would limit pain and improve quality of life and function in patients with KOA as compared with those receiving no exercise intervention.

\section{METHODS}

\section{Study Design}

The present study was a single-center, single-blind, randomized controlled trial performed with patients of the physiotherapy clinic of the Onofre Lopes University Hospital (Natal, Brazil). The study was carried out between June 2011 and November 2011 after approval by relevant local research ethics committees.

\section{Subjects and Eligibility}

We recruited patients aged above $18 \mathrm{yr}$ with symptomatic clinical diagnosis of chronic KOA (based on the criteria of the American College of Rheumatology) and moderate to very severe knee pain according to the Lequesne algofunctional index (scores ranging from 5 to 13). Patients were referred from rheumatologists and were using stable doses of anti-inflammatory drugs. All individuals had experienced pain within the last year in or around the knee occurring on most days for at least 3 mo. Exclusion criteria were as follows: cognitive dysfunction, previous participation in a similar rehabilitation program, medical contraindication to mild to moderate physical activity, other causes of pain in the lower limb, refusal to continue the study, and two consecutive or three nonconsecutives absences.

\section{Randomization}

A computer-based random number sequence was generated by a volunteer who was not involved in eligibility assessment, data collection, or analysis. The allocations were concealed from the investigators by enrolling participants in sequentially numbered opaque, sealed envelopes. Participants were randomized in a 1:1 allocation to the intervention group (IG) or the control group (CG). Owing to the nature of the interventions, it was not possible to blind the staff administering them.

\section{Intervention}

Prior to randomization, the patients participated in a self-management program that consisted of a $90 \mathrm{~min}$ lecture containing general orientation about osteoarthritis. The purpose of providing a self-management class prior to randomization was to ensure greater adhesion and providing information about the study and KOA. After randomization, CG participants received booster educational information about the disease and how to improve quality of life and function through leaflets for each of the $8 \mathrm{wk}$, but no additional intervention. The themes of the leaflets were the same basic information given to IG participants during the same period (more detail later). The IG participated in a group rehabilitation program that consisted of $60 \mathrm{~min}$ sessions performed twice a week for a period of $8 \mathrm{wk}$ and supervised by four students of physiotherapy duly trained. This program included educational aspects about KOA 
(15 min) followed by several physical activities (45 min). The education sessions were adapted from Hurley et al. [13] and consisted of seminars and discussion groups, which had the following themes: aims and objectives of the program; identification of personal objectives and recognition of individual functional capabilities; weight control and constituents of a healthy diet, including possible benefits of omega-3; explanation of pain perceptions and biopsychosocial model of pain; nonpharmacological procedures of pain management and use of ice and heat when appropriate; and home exercise and home relaxation techniques. Physical activities included the following: warm-up for $10 \mathrm{~min}$ with a stationary bike and stretching; exercises for the strength of the lower and upper limbs; body mobility, functional, and balance exercises; and relaxation (Table 1). The load used for the strength training was defined based on a 10-repetition maximum test rather than a 1-repetition maximum test to prevent damage caused by an excessive muscle contraction. Fifty to sixty percent of the estimated maximum load was used [6]. Exercise intensity was maintained at a moderate level of perceived effort according to recommendations for individuals with disabling conditions by the American College of Sports Medicine and American Heart Association [16]. We used the Borg scale to assess the level of effort during and after each exercise and to adjust exercise progression [17]. If the IG participants reported ease in performing exercises or were able to add two extra repetitions to the set, the load was increased in the next session. Self-reported pain was monitored before and immediately after the intervention sessions by using the visual analog scale, where 0 indicates no pain and 10 indicates the worst possible pain. Levels from 0 to 5 were defined as acceptable pain [18]. If participants had self-reported pain greater than 5 or were not able to complete a training session, they were instructed to stop the session.

\section{Clinical Evaluation}

The Lequesne algofunctional index was used as a tool for primary outcome assessment (the questionnaire's total score). This questionnaire is a widely used instrument to measure severity of KOA, which was translated into Portuguese and validated in 2006 [19]. It comprises three dimensions, including pain (5 items), maximum walking distance ( 2 items), and activities of daily living (function subdomain, 8 items). Each dimension has a maximum total score of 8 . The total Lequesne score ranges from 0 to 24 , and a higher score indicates a worse function [20].

Secondary outcome measures were assessed by performance tests and the 36-Item Short Form Health Survey (SF-36). This survey is a commonly used measure of general health and related quality of life that consists of eight subscales: physical function, role physical, bodily pain, general health, vitality, social functioning, role emotional, and mental health [21]. The performance tests used in this study were the chair-stand, sit-and-reach, timed up-and-go, and 6-minute walk tests. The chairstand test can be used to monitor lower-body strength since it quantifies the maximum number of chair-stands completed within $30 \mathrm{~s}$ [22]. The sit-and-reach test is a valid and common measure of flexibility for lower back and hamstring muscles [23]. Finally, the timed up-and-go and 6-minute walk tests are timed mobility tests commonly used to evaluate functional performance [24]. All questionnaires and tests were conducted before and after the $8 \mathrm{wk}$ intervention by two blinded physiotherapists.

\section{Data Analysis}

Data analyses were conducted with SPSS version 20.0 (IBM Corporation; Armonk, New York). Independent $t$-tests were used to compare the data for the two randomized groups at baseline. Differences in week 8 outcomes between the groups were assessed by analysis of covariance (ANCOVA), using the pretest results as the covariate, in a complete case analysis. ANCOVA has been recommended to increase the precision of estimates and the statistical power while also reducing the sample size requirements [25-26]. As stated by White et al., complete case analysis is appropriate for randomized trials with pretest-posttest designs [27]. They demonstrate that including baseline values in a regression model of the posttest outcomes (ANCOVA's principle) account for dropout bias [27]. This is the best approach for our study since we faced informative and completely at-random dropouts (summary of the dropout reasons is included in the "Results" section). At the review process, this study underwent positive changes. Some of these included the decision to use the ANCOVA as the primary analysis, such that reporting our previous sample size estimation (or a new one) would be pointless. Thus, we were cautious by evaluating whether the ANCOVA (with $\alpha=0.05$ and power $=0.8$ ) could still ensure the effect sizes obtained, given the final sample size. By conducting a sensitivity power analysis with $\mathrm{G}^{*}$ power software (version 3.1.6) [28], we found a minimum detectable effect (MDE) 
JRRD, Volume 52, Number 3, 2015

Table 1.

Description of exercises and other activities performed by intervention group.

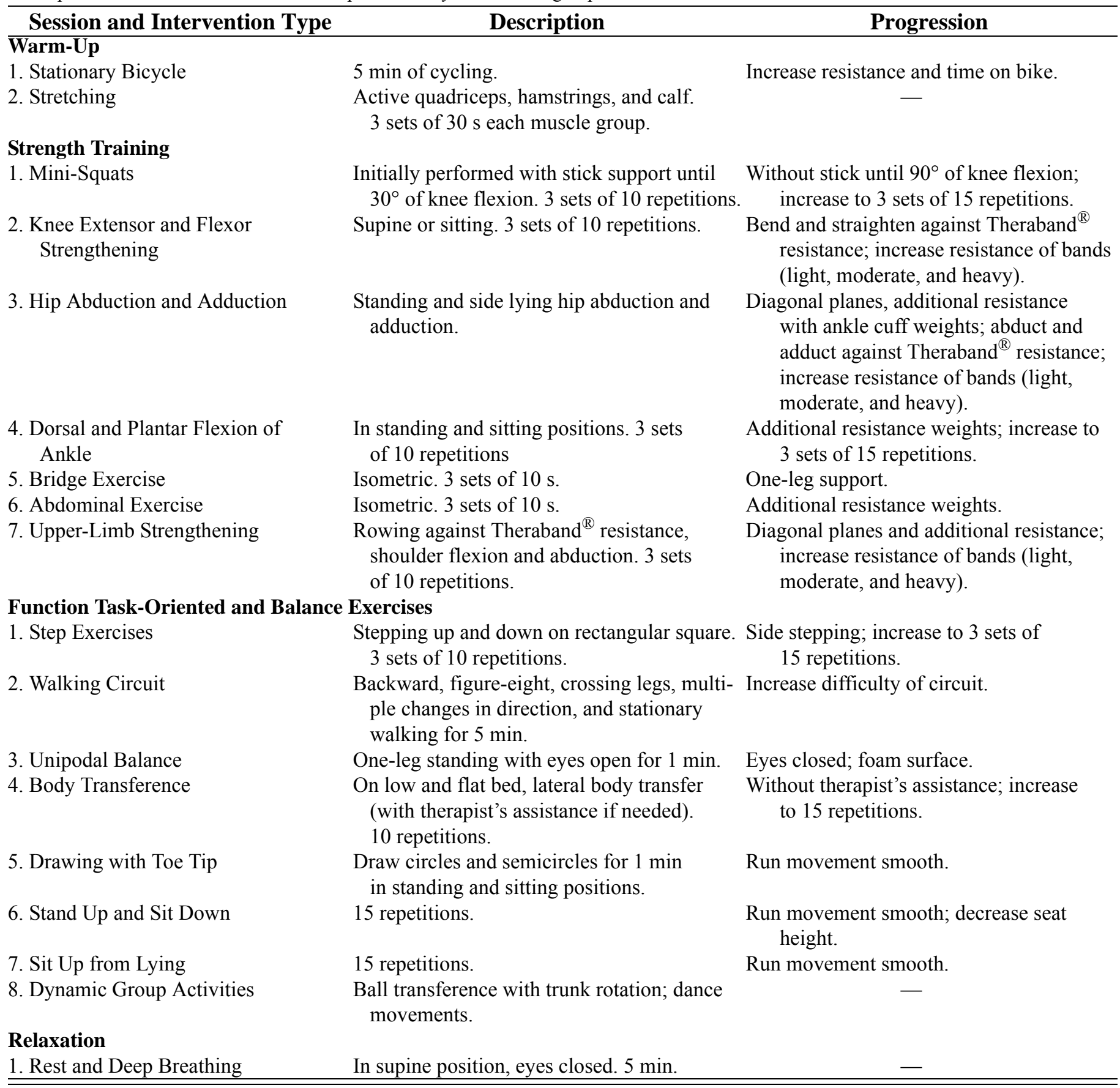

of 0.22 in absolute values of partial eta squared $\left(\eta_{p}^{2}\right)$. Effect size threshold for minimum clinically important difference (MCID) was used to supplement the conventional statistical interpretation of the results. MCID was defined by $\eta_{p}^{2}$ equal to 0.01 (equivalent to a Cohen $d$ of 0.2 ) based on a commonly accepted standard for small effects. Small, medium, and large effects correspond to values of $\eta_{p}^{2}$ of $0.01,0.06$, and 0.14 , respectively [29]. A 90 percent (1$2 \alpha$ ) confidence interval (CI) for $\eta_{p}^{2}$ was also reported since the effect size is an upper-tailed (0 to 1) parameter [30]. $p$-Values $<0.05$ were regarded as significant. Data are expressed as mean \pm standard deviation or $n$ (percent). 


\section{RESULTS}

A total of 52 patients were identified and selected for participation in this study. At the baseline assessment, 11 patients were excluded due to uncontrolled hypertension $(n=4)$ and Lequesne index scores higher than $13(n=5)$ and lower than $5(n=2)$. The remaining 41 participants were randomized to the IG (19 participants) or CG (22 participants). One erroneous inclusion occurred in favor of the CG. During the $8 \mathrm{wk}$ trial, four IG participants declined to take part in this work due to absences $(n=1)$ or personal reasons $(n=3)$. In parallel, seven CG participants dropped out due to health problems $(n=2)$ or personal reasons $(n=5)$. Displaying a "completely at random" mechanism, the specific dropout reasons were reported as absences ( 1 due to problems with transportation), health problems ( 1 due to influenza virus infection and 1 due to muscle injury from slipping during home cleaning) and personal reasons ( 2 due to incompatible timetable, 1 due to moving away from study area, 1 due to heavy alcohol use, 1 due to obtaining new employment, 1 due to taking care of grandchildren, 1 due to emotional indisposition after familial conflict, and 1 due to receiving limited support from family). At the end of the study, 30 (73\%) of the 41 participants completed the trial (15 in each group). The dropout rates were 21 and 32 percent for the IG and CG, respectively. Figure 1 outlines the overall structure of the study.

\section{Basic Characteristics}

Table 2 outlines the demographic and clinical characteristics of patients according to age, weight, height, body mass index, sex, education level, comorbidities, and lifestyle. In general, the patients of both groups were homogeneous in these characteristics.

\section{Comparisons Between Groups at Baseline}

Prior to any intervention, the groups had no significant differences with respect to main assessed outcomes, including Lequesne index total score and pain, distance, and function subdomains; SF-36 physical function, role physical, bodily pain, general health, vitality, social function, role emotional, and mental health subdomains; and performance assessed by the chair-stand, sit-and-reach, timed up-and-go, and 6-minute walk tests (all $p>0.05$ ) (Table 3).

\section{Comparisons Between Groups at Week 8}

At the end of trial, when controlling for baseline values, the IG exhibited significant differences compared with the CG $(p<0.05)$ regarding most of the outcomes, except the Lequesne distance subdomain, SF-36 social function and mental health subdomains, and sit-andreach test $(p>0.05)$ (Table 3). All these differences consisted of positive responses of the IG to the group rehabilitation program, as more precisely indicated by the week 8 adjusted outcome means and 95 percent CI of both groups, according to the ANCOVA model. No worsening effects were verified in both groups of patients (Table 4).

\section{Effect Sizes}

Figure 2 displays a summary of the 8 wk treatment outcomes with regard to clinically important benefits of the group rehabilitation program. Clinically important benefits (i.e., an effect size greater than 0.01) were found for most outcomes that were statistically significant with ANCOVA. These benefits may be uncertain for the Lequesne index function subdomain and SF-36 bodily pain and general health subdomains, whose absolute effect size values do not reach the MDE. When ANCOVA exhibited nonsignificant results in the assessed outcomes, the values of effect sizes were small and include 0 on CI.

\section{DISCUSSION}

The results of the present study showed that the group rehabilitation program was efficacious to improve pain, quality of life, and functionality of patients with KOA as compared with those who only received health educational support. There were significant improvements in the Lequesne index of pain and daily activities subdomains as well as the total score. Furthermore, IG participants improved in virtually all the functional performance tests. The rehabilitation program also produced significant improvements in the SF-36 physical function, role physical, vitality, and role emotional subdomains. In general, these data indicate that IG participants had less pain and functional disabilities, thus achieving a better outcome on quality of life.

Exercise is a key component of the conservative nonpharmacological treatment for patients with osteoarthritis, and it may decrease pain sensitivity [31] and improve 


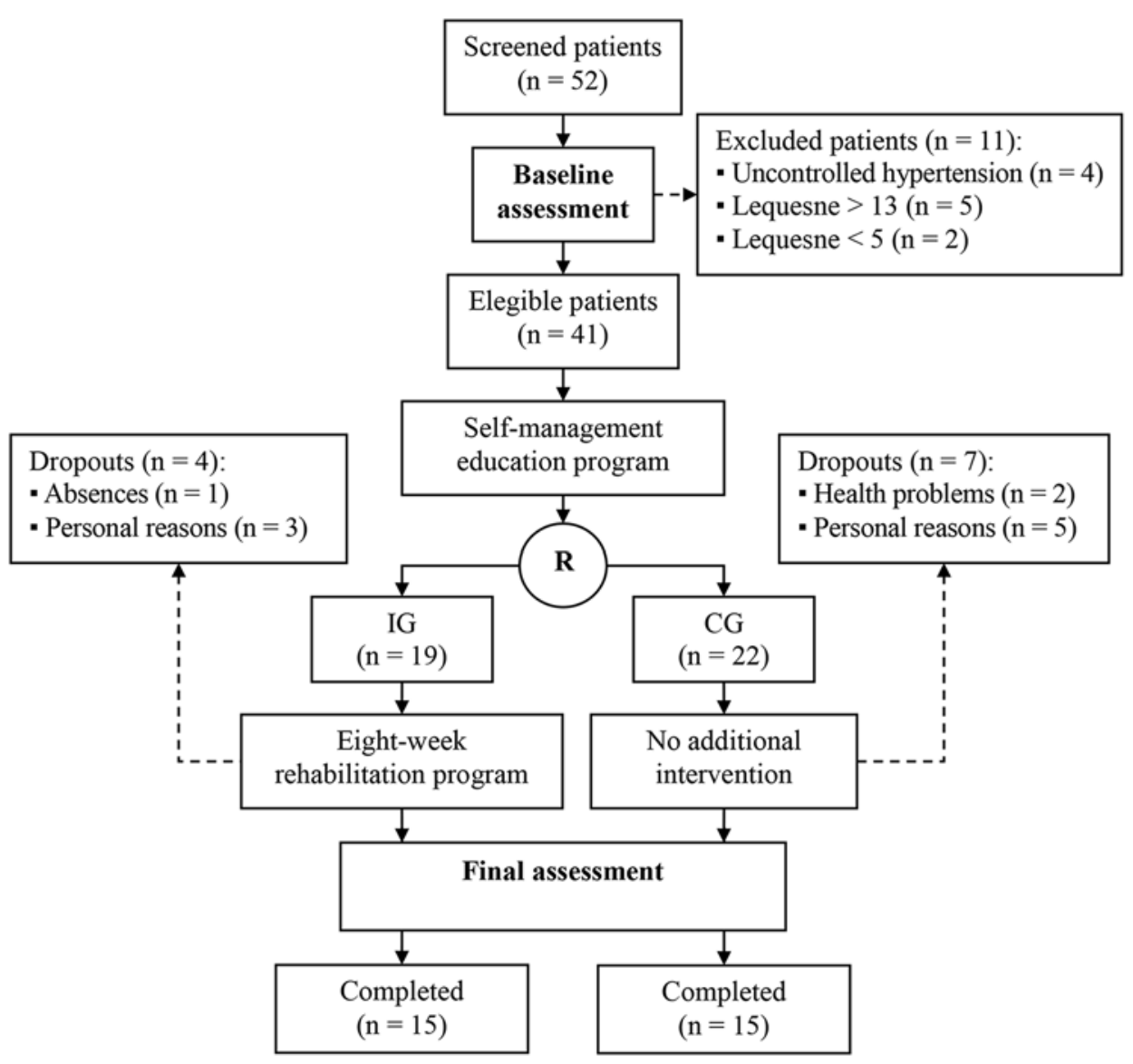

Figure 1.

Flowchart of study participants. CG = control group, IG = intervention group, $\mathrm{R}=$ randomization.

muscle strength, joint range of motion, proprioception, balance, and cardiovascular fitness [32-34] without any large adverse effects or costs. On the other hand, inappropriate exercises can exacerbate knee pain and damage the joint structure. We designed an integrated rehabilitation program composed of effective, simple, and safe exercises that are commonly used and reproduced in the clinical practice for KOA and hip osteoarthritis management $[7,15]$ associated with a self-management education program.

Considering recommendations for exercise prescription [9], our program consisted mainly of lower-limb strength and functional exercises but included some stretching and aerobic exercise. We did not include hydrotherapy, electrotherapy, and strength training exercises that required infrastructure and specific equipment. This study focused on patients with moderate to very severe levels of KOA, according to the Lequesne index.
None of the subjects reported major difficulties in performing the exercises.

It must be highlighted that group rehabilitation programs allow a greater number of participants and gives the opportunity to more easily sort out the most serious cases for individual care. In fact, researchers have shown that a group rehabilitation program is able to reduce public health costs $[9,35]$ and may be as effective as individual treatment [13].

It is well known that KOA presents a natural progression of pain and reduced functional capacity [36-37]. CG participants maintained pain and function level over this 8 wk study (data not shown). This may be due to the short period ( $8 \mathrm{wk}$ ) of intervention, possibly insufficient to cause changes in the levels of pain and function. Conversely, we cannot rule out influence of the self-management education program provided at the beginning of the study and during the following weeks by means of leaflets. This notion is 
Table 2.

Demographic and clinical characteristics at baseline.

\begin{tabular}{lcc}
\hline \multicolumn{1}{c}{ Item } & $\begin{array}{c}\text { Intervention } \\
\text { Group } \\
(\boldsymbol{n}=\mathbf{1 5})\end{array}$ & $\begin{array}{c}\text { Control } \\
\text { Group } \\
(\boldsymbol{n}=\mathbf{1 5})\end{array}$ \\
\hline Age, yr (mean \pm SD) & $57 \pm 6.01$ & $60 \pm 7.76$ \\
Weight, kg (mean \pm SD) & $72.22 \pm 11.43$ & $69.43 \pm 10.57$ \\
Height, m (mean \pm SD) & $1.57 \pm 0.09$ & $1.54 \pm 0.10$ \\
Body Mass Index (mean \pm SD) & $29.37 \pm 4.10$ & $29.29 \pm 5.00$ \\
Sex (\%) & & \\
Male & $2(13.33)$ & $2(13.33)$ \\
Female & $13(86.87)$ & $13(86.67)$ \\
Education Level (\%) & $1(6.67)$ & $1(6.67)$ \\
Illiteracy & $4(26.67)$ & $8(53.33)$ \\
Elementary School & $9(60.00)$ & $5(33.33)$ \\
High School & $1(6.67)$ & $1(6.67)$ \\
College & & \\
Comorbidity (\%) & $1(6.67)$ & $2(13.33)$ \\
Diabetes Mellitus & $11(73.33)$ & $7(46.67)$ \\
Hypertension & $1(6.67)$ & $1(6.67)$ \\
Hypercholesterolemia & & \\
Lifestyle (\%) & $8(53.33)$ & $10(66.67)$ \\
Sedentary & $0(0.00)$ & $1(6.67)$ \\
Alcoholism & $0(0.00)$ & $1(6.67)$ \\
Smoker & $2(13.33)$ & $2(13.33)$ \\
Former Smoker & & \\
\hline SD $=$ standard deviation. & & \\
\hline \hline & & \\
\hline
\end{tabular}

shared by other works and suggests that general orientation and health education are effective to maintain and/or increase functionality and control pain in subjects with KOA [35,38]. Nonetheless, Skou et al. suggest that group education must be reinforced with exercises for better results in individuals with osteoarthritis [39].

Our sample consisted of individuals with lower educational levels than other works [40-41]. The lower educational level of the whole sample may have influenced some outcomes related to self-perception of health status. It is well known that socioeconomic status is the main factor that influences self-perception of health status, especially among the elderly [42]. Indeed, people with lower educational levels perceive their health as worse than those with university diplomas [43]. It is possible that the lower educational level of the participants may have been partially responsible for the limited psychosocial improvements.

It is noteworthy to mention that we did not detect improvements in the sit-and-reach test in both groups. The sit-and-reach test is a common measure of flexibility of the hamstrings and lower back muscles. The rehabili- tation program contained stretching exercises for the major muscle groups of the lower limbs, as recommended in the literature $[6,44]$. However, it is possible that this ability has not improved due to the duration (about $5 \mathrm{~min}$ ) and frequency ( 2 times/wk) of the stretching exercises, which are below the recommended levels for people with osteoarthritis [45-46].

In this work, we employed the ANCOVA to assess differences between the groups at the end of the trial. The covariate adjustment for baseline values avoided possible interference in the results due to variation of the sample, which was present since the initial assessment. We can ensure that 8 wk effects on the parameters where the groups showed differences were due to the rehabilitation program. The presented data characterize precise intervention effects, as indicated for obtained $\eta_{p}^{2}$ and its 90 percent CIs. Like the $\eta_{p}^{2}$ estimate, the corresponding CIs cannot be less than 0 . Since the CI represents the precision with which we are able to report the effect size, employing a confidence coefficient of 90 percent is an appropriate way to verify the size of an effect [30]. It must be pointed out that some CIs covered small or medium parameter values despite a corresponding large $\eta_{p}^{2}$ absolute value. Lower confidence bounds, including values from 0.01 to 0.05 , were observed for Lequesne index total score and pain and function subdomains and SF-36 bodily pain, general health, and role emotional subdomains. Thus, a small effect could not be refuted for these outcomes. In the case of the $\eta^{2}$ p values for SF-36 physical function and vitality subdomains and chair-stand and 6minute walk tests, its corresponding lower confidence bounds included values from 0.07 to 0.13 , so that a medium effect could not be rejected for these outcomes. Very large effects were found for the SF-36 role physical subdomain and for the timed up-and-go test. For these, all effect size values were accompanied by lower confidence bounds above 0.14 . As might be expected from an exercise-based rehabilitation program, these results indicate a greater magnitude of improvement on the physical performance-related outcomes, followed by minor improvements on psychosocial-related outcomes. In general, clinically important benefits were found for all the outcomes that were statistically significant with ANCOVA, without noticeable harm for the studied participants. From a conservative point of view, however, we are not certain about clinically important benefits for the Lequesne index function subdomain or SF-36 bodily pain and general health subdomains, since their effect sizes (in absolute 
JRRD, Volume 52, Number 3, 2015

Table 3.

Comparison between intervention group (IG) $(n=15)$ and control group (CG) $(n=15)$ at baseline and at end of trial (week 8).

\begin{tabular}{|c|c|c|c|c|c|c|}
\hline \multirow{2}{*}{ Outcome } & \multicolumn{3}{|c|}{ Baseline } & \multicolumn{3}{|c|}{ Week 8} \\
\hline & IG & $\mathbf{C G}$ & p-Value ${ }^{*}$ & IG & $\mathbf{C G}$ & $p$-Value ${ }^{\dagger}$ \\
\hline \multicolumn{7}{|c|}{ Lequesne Index (mean \pm SD) } \\
\hline Pain & $4.93 \pm 1.33$ & $4.47 \pm 1.46$ & 0.37 & $2.60 \pm 1.55$ & $4.00 \pm 1.56$ & 0.009 \\
\hline Distance & $1.13 \pm 0.83$ & $1.07 \pm 0.88$ & 0.83 & $0.60 \pm 0.51$ & $0.73 \pm 0.80$ & 0.51 \\
\hline Function & $3.57 \pm 1.08$ & $3.23 \pm 1.53$ & 0.49 & $2.30 \pm 1.36$ & $3.13 \pm 1.45$ & 0.02 \\
\hline Total & $9.63 \pm 1.91$ & $8.77 \pm 3.18$ & 0.38 & $5.50 \pm 2.98$ & $7.87 \pm 3.48$ & 0.009 \\
\hline \multicolumn{7}{|l|}{ SF-36 $($ mean \pm SD $)$} \\
\hline Physical Function & $39.67 \pm 15.86$ & $47.67 \pm 29.99$ & 0.37 & $65.33 \pm 11.57$ & $51.33 \pm 21.25$ & 0.001 \\
\hline Role Physical & $30.00 \pm 35.61$ & $28.33 \pm 31.15$ & 0.89 & $88.33 \pm 20.85$ & $35.00 \pm 39.87$ & $<0.001$ \\
\hline Bodily Pain & $44.47 \pm 11.78$ & $41.27 \pm 17.88$ & 0.57 & $57.60 \pm 12.48$ & $42.80 \pm 21.52$ & 0.03 \\
\hline General Health & $52.40 \pm 24.50$ & $52.07 \pm 20.78$ & 0.97 & $69.00 \pm 18.59$ & $55.27 \pm 17.86$ & 0.02 \\
\hline Vitality & $56.00 \pm 19.20$ & $60.00 \pm 12.54$ & 0.51 & $72.00 \pm 15.56$ & $58.33 \pm 16.22$ & 0.003 \\
\hline Social Function & $86.67 \pm 13.75$ & $87.50 \pm 16.37$ & 0.88 & $91.67 \pm 12.20$ & $90.83 \pm 13.75$ & 0.83 \\
\hline Role Emotional & $53.27 \pm 43.33$ & $51.00 \pm 39.65$ & 0.88 & $86.67 \pm 30.37$ & $53.20 \pm 32.99$ & 0.006 \\
\hline Mental Health & $71.20 \pm 21.97$ & $57.87 \pm 15.03$ & 0.06 & $75.20 \pm 18.77$ & $61.07 \pm 20.92$ & 0.40 \\
\hline \multicolumn{7}{|c|}{ Performance Test (mean \pm SD) } \\
\hline Chair-Stand Test & $10.07 \pm 2.49$ & $11.27 \pm 2.89$ & 0.23 & $14.07 \pm 2.52$ & $11.33 \pm 3.24$ & $<0.001$ \\
\hline Sit-and-Reach Test & $20.81 \pm 9.31$ & $18.33 \pm 6.44$ & 0.40 & $22.27 \pm 7.83$ & $17.78 \pm 7.83$ & 0.19 \\
\hline Timed Up-and-Go Test & $8.70 \pm 1.48$ & $9.25 \pm 2.76$ & 0.50 & $7.17 \pm 0.94$ & $9.22 \pm 1.89$ & $<0.001$ \\
\hline 6-Minute Walk Test & $409.77 \pm 48.11$ & $417.20 \pm 67.90$ & 0.73 & $485.47 \pm 57.99$ & $435.07 \pm 64.40$ & 0.001 \\
\hline
\end{tabular}

Table 4.

Posttest adjusted mean and 95 percent confidence interval (CI) of variables in intervention group (IG) and control group (CG), using pretest as covariate.

\begin{tabular}{|c|c|c|c|c|}
\hline \multirow{2}{*}{ Outcome } & \multicolumn{2}{|c|}{ IG $(n=15)$} & \multicolumn{2}{|c|}{ CG $(n=15)$} \\
\hline & Mean \pm SE & $95 \% \mathrm{CI}$ & Mean \pm SE & $95 \% \mathrm{CI}$ \\
\hline \multicolumn{5}{|l|}{ Lequesne Index } \\
\hline Pain ${ }^{*}$ & $2.52 \pm 0.39$ & $1.72-3.33$ & $4.08 \pm 0.39$ & $3.28-4.88$ \\
\hline Distance & $0.59 \pm 0.16$ & $0.26-0.93$ & $0.74 \pm 0.16$ & $0.41-1.08$ \\
\hline Function $^{*}$ & $2.19 \pm 0.29$ & $1.59-2.79$ & $3.24 \pm 0.29$ & $2.64-3.84$ \\
\hline Total $^{*}$ & $5.22 \pm 0.73$ & $3.72-6.72$ & $8.15 \pm 0.73)$ & $6.65-9.65$ \\
\hline \multicolumn{5}{|l|}{ SF-36 } \\
\hline Physical Function* & $67.34 \pm 3.23$ & $60.72-73.96$ & $49.33 \pm 3.23$ & $42.71-55.95$ \\
\hline Role Physical $^{*}$ & $88.19 \pm 8.23$ & $71.31-105.07$ & $35.14 \pm 8.23$ & $18.26-52.03$ \\
\hline Bodily Pain ${ }^{*}$ & $56.61 \pm 3.92$ & $48.56-64.66$ & $43.79 \pm 3.92$ & $35.74-51.84$ \\
\hline General Health $^{*}$ & $68.92 \pm 3.74$ & $61.24-76.59$ & $55.35 \pm 3.74$ & $47.67-63.03$ \\
\hline Vitality $^{*}$ & $73.16 \pm 3.38$ & $66.22-80.10$ & $57.17 \pm 3.38$ & $50.23-64.11$ \\
\hline Social Function & $91.76 \pm 3.30$ & $85.00-98.52$ & $90.74 \pm 3.30$ & $83.98-97.50$ \\
\hline Role Emotional $^{*}$ & $86.36 \pm 7.79$ & $70.37-102.35$ & $53.51 \pm 7.79$ & $37.52-69.50$ \\
\hline Mental Health & $70.74 \pm 4.18$ & $62.17-79.32$ & $65.52 \pm 4.18$ & $56.95-74.10$ \\
\hline \multicolumn{5}{|l|}{ Performance Test } \\
\hline Chair-Stand Test ${ }^{*}$ & $14.46 \pm 0.61$ & $13.21-15.72$ & $10.94 \pm 0.61$ & $9.68-12.19$ \\
\hline Sit-and-Reach Test & $21.42 \pm 1.46$ & $18.41-24.42$ & $18.64 \pm 1.46$ & $15.64-21.64$ \\
\hline Timed Up-and-Go Test ${ }^{*}$ & $7.28 \pm 0.32$ & $6.64-7.93$ & $9.11 \pm 0.32$ & $8.46-9.76$ \\
\hline 6-Minute Walk Test ${ }^{*}$ & $488.47 \pm 10.16$ & $467.62-509.33$ & $432.06 \pm 10.16$ & $411.21-452.92$ \\
\hline
\end{tabular}




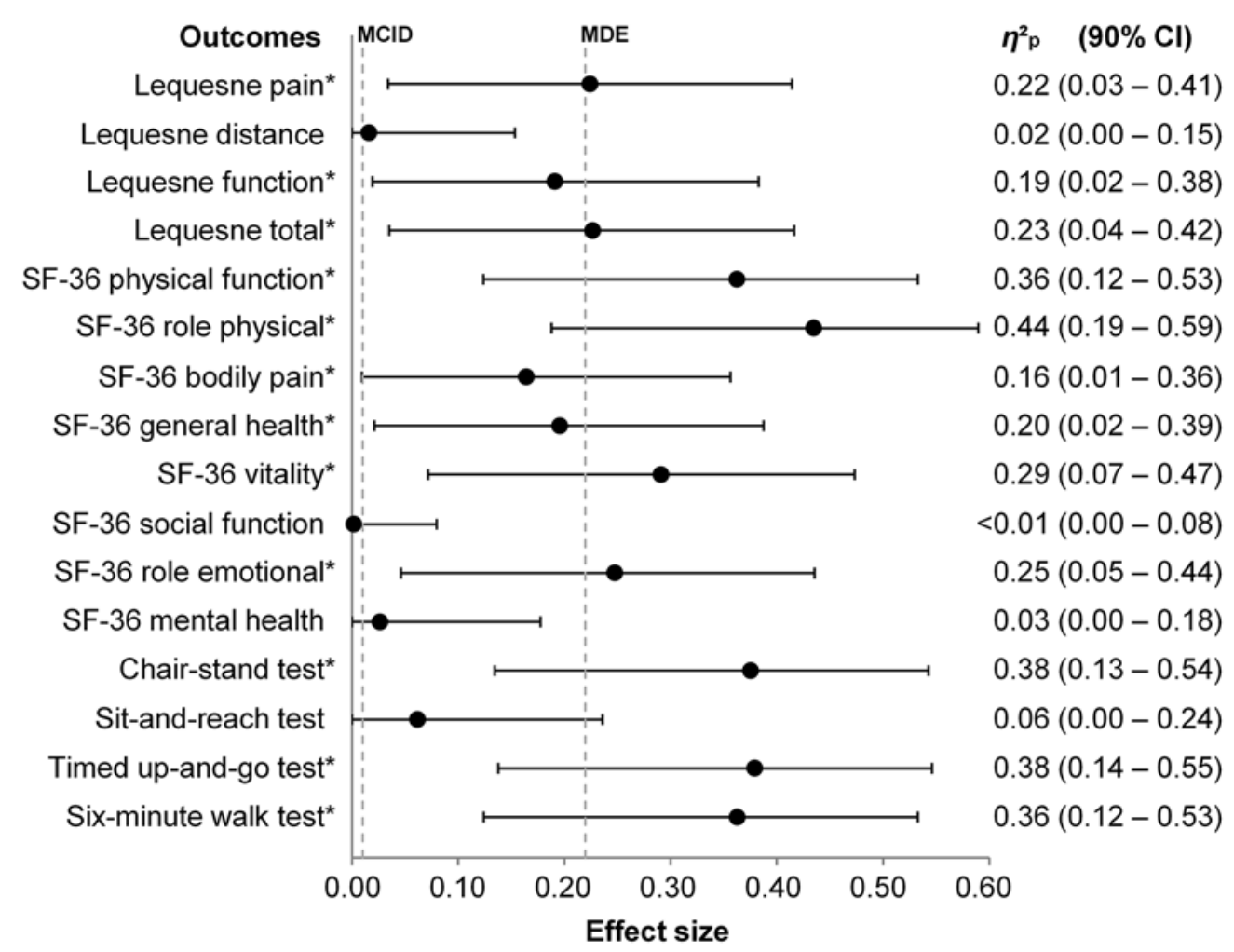

Figure 2.

Graphical summary of intervention effects. Results are presented as effect size $\left(\eta^{2} p\right)$ and 90 percent confidence interval $(\mathrm{Cl})$. $\mathrm{MCID}=$ minimum clinically important difference, MDE = minimum detectable effect, SF-36 = 36-Item Short Form Health Survey.

values) were inferior to the $\operatorname{MDE}\left(\eta_{p}^{2}=0.22\right)$. Thus, the set of results presented here allows us to infer equivalent therapeutic effects for a population with KOA with similar characteristics to the sample of the study.

This study has some limitations. We focused only on short-term effects of the $8 \mathrm{wk}$ rehabilitation program. The generalization of our findings is limited since it is not known whether the positive effects will be long-term or not. In a recent work, Pisters et al. showed that two different physical therapy interventions were effective longterm for patients with KOA and hip osteoarthritis, improved pain and physical function, and showed that patients' global assessment persisted for up to $60 \mathrm{mo}$ [47]. In a previous systematic review, the same group found that the positive posttreatment effects of exercise therapy on pain and physical function in patients with KOA or hip osteoarthritis were not sustained in the longterm [7]. Conversely, the long-term effectiveness for patients' global assessment persisted. Also, they found that booster sessions performed periodically could influence maintenance of beneficial posttreatment effects on pain and physical function in the long-term. In this way, it is reasonable to believe that the group rehabilitation program presented here may improve adherence via eventual booster sessions that may facilitate control of progression of the disease.

It is well known that exercises may increase muscle strength, agility and speed, dynamic balance, and aerobic capacity [22]. Notwithstanding, we did not perform quantitative measurements of muscle strength, and future studies should investigate these issues to determine the efficacy and intensity of training strength.

Finally, CG participants only received health information through leaflets after randomization and, therefore, received less attention than IG participants. Thus, we cannot exclude the influence of the Hawthorne Effect [48]. However, this approach has been carried out before for a CG via an arthritis self-management book and no change in pain, self-efficacy, disability, or mental health was observed [49]. Moreover, the leaflets contained the same educational instructions presented to the IG during the 8 wk rehabilitation program. 


\section{CONCLUSIONS}

This research establishes a simple and evidencebased integrated group rehabilitation program consisting of exercise and a self-management program for patients with KOA. From the data gathered in this study, patients with moderate to very severe KOA who participated in this 8 wk study had alleviated pain and exhibited superior aspects of quality of life and functional outcomes compared with the CG. This rehabilitation protocol is presented as an acceptable option for KOA rehabilitation in clinical practice and research.

\section{ACKNOWLEDGMENTS}

\author{
Author Contributions: \\ Study concept and design: W. H. Brito Vieira. \\ Acquisition of data: F. E. de Melo, Í. L. Pinheiro, M. M. do Amaral, \\ V. V. Caldas. \\ Analysis and interpretation of data: W. H. Brito Vieira, F. S. da Silva, \\ B. J. Abreu. \\ Drafting of manuscript: W. H. Brito Vieira, F. S. da Silva, B. J. Abreu. \\ Critical revision of manuscript for important intellectual content: \\ W. H. Brito Vieira, F. S. da Silva, B. J. Abreu. \\ Statistical analysis: F. S. da Silva. \\ Study supervision: W. H. Brito Vieira. \\ Patient recruitment: F. E. de Melo, Í. L. Pinheiro, M. M. do Amaral, \\ V. V. Caldas.
}

Financial Disclosures: The authors have declared that no competing interests exist.

Funding/Support: This work was unfunded at the time of manuscript preparation.

Additional Contributions: The authors wish to thank all patients participating in the study.

Institutional Review: This work was performed in accordance with the Declaration of Helsinki, and the protocol was approved by the Federal University of Rio Grande do Norte Research Ethics Committee. All participants provided written informed consent to participate in the study, which was approved by the Federal University of Rio Grande do Norte Research Ethics Committee (protocol 530/11, CAAE: 0006.0.294.0000-11).

Participant Follow-Up: The authors plan to inform participants regarding the results of the study, and the publication will appear on the institutional Web site.

\section{REFERENCES}

1. Alkan BM, Fidan F, Tosun A, Ardıçoğlu O. Quality of life and self-reported disability in patients with knee osteoarthritis. Mod Rheumatol. 2014;24(1):166-71.
[PMID:24261774]

http://dx.doi.org/10.3109/14397595.2013.854046

2. Sun BH, Wu CW, Kalunian KC. New developments in osteoarthritis. Rheum Dis Clin North Am. 2007;33(1): 135-48. [PMID: 17367697]

http://dx.doi.org/10.1016/j.rdc.2006.12.003

3. Jordan KM, Arden NK, Doherty M, Bannwarth B, Bijlsma JW, Dieppe P, Gunther K, Hauselmann H, Herrero-Beaumont G, Kaklamanis P, Lohmander S, Leeb B, Lequesne M, Mazieres B, Martin-Mola E, Pavelka K, Pendleton A, Punzi L, Serni U, Swoboda B, Verbruggen G, Zimmerman-Gorska I, Dougados M; Standing Committee for International Clinical Studies Including Therapeutic Trials ESCISIT. EULAR Recommendations 2003: an evidence based approach to the management of knee osteoarthritis: Report of a Task Force of the Standing Committee for International Clinical Studies Including Therapeutic Trials (ESCISIT). Ann Rheum Dis. 2003;62(12):1145-55. [PMID:14644851]

http://dx.doi.org/10.1136/ard.2003.011742

4. Messier SP, Loeser RF, Miller GD, Morgan TM, Rejeski WJ, Sevick MA, Ettinger WH Jr, Pahor M, Williamson JD. Exercise and dietary weight loss in overweight and obese older adults with knee osteoarthritis: The Arthritis, Diet, and Activity Promotion Trial. Arthritis Rheum. 2004; 50(5):1501-10. [PMID:15146420]

http://dx.doi.org/10.1002/art.20256

5. Holden MA, Nicholls EE, Young J, Hay EM, Foster NE. Role of exercise for knee pain: What do older adults in the community think? Arthritis Care Res (Hoboken). 2012; 64(10):1554-64. [PMID:22511582]

http://dx.doi.org/10.1002/acr.21700

6. Roddy E, Zhang W, Doherty M, Arden NK, Barlow J, Birrell F, Carr A, Chakravarty K, Dickson J, Hay E, Hosie G, Hurley M, Jordan KM, McCarthy C, McMurdo M, Mockett S, O'Reilly S, Peat G, Pendleton A, Richards S. Evidence-based recommendations for the role of exercise in the management of osteoarthritis of the hip or knee- the MOVE consensus. Rheumatology (Oxford). 2005;44(1): 67-73. [PMID:15353613] http://dx.doi.org/10.1093/rheumatology/keh399

7. Pisters MF, Veenhof C, van Meeteren NL, Ostelo RW, de Bakker DH, Schellevis FG, Dekker J. Long-term effectiveness of exercise therapy in patients with osteoarthritis of the hip or knee: A systematic review. Arthritis Rheum. 2007;57(7):1245-53. [PMID:17907210] http://dx.doi.org/10.1002/art.23009

8. Hurley MV, Walsh N, Bhavnani V, Britten N, Stevenson F. Health beliefs before and after participation on an exercised-based rehabilitation programme for chronic knee pain: Doing is believing. BMC Musculoskelet Disord. 2010;11:31. [PMID:20149236] http://dx.doi.org/10.1186/1471-2474-11-31 
9. Bennell KL, Hinman RS. A review of the clinical evidence for exercise in osteoarthritis of the hip and knee. J Sci Med Sport. 2011;14(1):4-9. [PMID:20851051] http://dx.doi.org/10.1016/i.jsams.2010.08.002

10. Subramaniam V, Stewart MW, Smith JF. The development and impact of a chronic pain support group: A qualitative and quantitative study. J Pain Symptom Manage. 1999; 17(5):376-83. [PMID:10355217] http://dx.doi.org/10.1016/S0885-3924(99)00012-3

11. Tiffreau V, Mulleman D, Coudeyre E, Lefevre-Colau MM, Revel M, Rannou F. The value of individual or collective group exercise programs for knee or hip osteoarthritis. Clinical practice recommendations. Ann Readapt Med Phys. 2007;50(9):741-46, 734-40. [PMID:17963971] http://dx.doi.org/10.1016/j.annrmp.2007.10.001

12. Fransen M, McConnell S, Bell M. Therapeutic exercise for people with osteoarthritis of the hip or knee. A systematic review. J Rheumatol. 2002;29(8):1737-45.

[PMID:12180738]

13. Hurley MV, Walsh NE, Mitchell HL, Pimm TJ, Patel A, Williamson E, Jones RH, Dieppe PA, Reeves BC. Clinical effectiveness of a rehabilitation program integrating exercise, self-management, and active coping strategies for chronic knee pain: A cluster randomized trial. Arthritis Rheum. 2007;57(7):1211-19. [PMID:17907147] http://dx.doi.org/10.1002/art.22995

14. Jessep SA, Walsh NE, Ratcliffe J, Hurley MV. Long-term clinical benefits and costs of an integrated rehabilitation programme compared with outpatient physiotherapy for chronic knee pain. Physiotherapy. 2009;95(2):94-102.

[PMID:19627690] http://dx.doi.org/10.1016/j.physio.2009.01.005

15. Uthman OA, van der Windt DA, Jordan JL, Dziedzic KS, Healey EL, Peat GM, Foster NE. Exercise for lower limb osteoarthritis: Systematic review incorporating trial sequential analysis and network meta-analysis. BMJ. 2013; 347:f5555. [PMID:24055922] http://dx.doi.org/10.1136/bmj.f5555

16. Nelson ME, Rejeski WJ, Blair SN, Duncan PW, Judge JO, King AC, Macera CA, Castaneda-Sceppa C. Physical activity and public health in older adults: Recommendation from the American College of Sports Medicine and the American Heart Association. Med Sci Sports Exerc. 2007; 39(8):1435-45. [PMID:17762378] http://dx.doi.org/10.1249/mss.0b013e3180616aa2

17. Borg G. Ratings of perceived exertion and heart rates during short-term cycle exercise and their use in a new cycling strength test. Int J Sports Med. 1982;3(3):153-58.

[PMID:7129724]

http://dx.doi.org/10.1055/s-2008-1026080
18. Thomeé R. A comprehensive treatment approach for patellofemoral pain syndrome in young women. Phys Ther. 1997;77(12):1690-1703. [PMID:9413448]

19. Marx FC, Oliveira LM, Bellini CG, Ribeiro MC. Translation and cultural validation of the Lequesne's algofunctional questionnaire for osteoarthritis of knee and hip for Portuguese language. Br J Rheumatol. 2006;46(4):253-60.

20. Lequesne MG. The algofunctional indices for hip and knee osteoarthritis. J Rheumatol. 1997;24(4):779-81.

[PMID:9101517]

21. Ware JE Jr, Sherbourne CD. The MOS 36-item short-form health survey (SF-36). I. Conceptual framework and item selection. Med Care. 1992;30(6):473-83. [PMID:1593914] http://dx.doi.org/10.1097/00005650-199206000-00002

22. Rikli RE, Jones CJ. Development and validation of criterion-referenced clinically relevant fitness standards for maintaining physical independence in later years. Gerontologist. 2013;53(2):255-67. [PMID:22613940] http://dx.doi.org/10.1093/geront/gns071

23. Muyor JM, Zemková E, Štefániková G, Kotyra M. Concurrent validity of clinical tests for measuring hamstring flexibility in school age children. Int J Sports Med. 2014;35(8): 664-69. [PMID:24424962]

http://dx.doi.org/10.1055/s-0033-1353217

24. Steffen TM, Hacker TA, Mollinger L. Age- and genderrelated test performance in community-dwelling elderly people: Six-Minute Walk Test, Berg Balance Scale, Timed Up \& Go Test, and gait speeds. Phys Ther. 2002;82(2): 128-37. [PMID:11856064]

25. Borm GF, Fransen J, Lemmens WA. A simple sample size formula for analysis of covariance in randomized clinical trials. J Clin Epidemiol. 2007;60(12):1234-38.

[PMID:17998077]

http://dx.doi.org/10.1016/j.jclinepi.2007.02.006

26. Egbewale BE, Lewis M, Sim J. Bias, precision and statistical power of analysis of covariance in the analysis of randomized trials with baseline imbalance: A simulation study. BMC Med Res Methodol. 2014;14:49. [PMID:24712304] http://dx.doi.org/10.1186/1471-2288-14-49

27. White IR, Horton N, Carpenter J. Complete-cases analysis is appropriate for randomised trials with pre-test-post-test designs. Psychiatry Res. 2009;168(3):268, author reply 269. [PMID:19556011]

http://dx.doi.org/10.1016/j.psychres.2009.01.003

28. Faul F, Erdfelder E, Lang AG, Buchner A. G*Power 3: A flexible statistical power analysis program for the social, behavioral, and biomedical sciences. Behav Res Methods. 2007;39(2):175-91. [PMID:17695343] http://dx.doi.org/10.3758/BF03193146

29. Cohen J. Statistical power analysis for the behavioural sciences. 2nd ed. Hillsdale (NJ): L. Erlbaum Associates; 1988. 
30. Steiger JH. Beyond the F test: Effect size confidence intervals and tests of close fit in the analysis of variance and contrast analysis. Psychol Methods. 2004;9(2):164-82. [PMID:15137887] http://dx.doi.org/10.1037/1082-989X.9.2.164

31. Burrows NJ, Booth J, Sturnieks DL, Barry BK. Acute resistance exercise and pressure pain sensitivity in knee osteoarthritis: A randomised crossover trial. Osteoarthritis Cartilage. 2014;22(3):407-14. [PMID:24418672] http://dx.doi.org/10.1016/j.joca.2013.12.023

32. Jan MH, Tang PF, Lin JJ, Tseng SC, Lin YF, Lin DH. Efficacy of a target-matching foot-stepping exercise on proprioception and function in patients with knee osteoarthritis. $\mathbf{J}$ Orthop Sports Phys Ther. 2008;38(1):19-25. [PMID:18357655] http://dx.doi.org/10.2519/jospt.2008.2512

33. Lange AK, Vanwanseele B, Fiatarone Singh MA. Strength training for treatment of osteoarthritis of the knee: A systematic review. Arthritis Rheum. 2008;59(10):1488-94. [PMID:18821647] http://dx.doi.org/10.1002/art.24118

34. Sekir U, Gür H. A multi-station proprioceptive exercise program in patients with bilateral knee osteoarthrosis: Functional capacity, pain and sensoriomotor function. A randomized controlled trial. J Sports Sci Med. 2005;4(4): 590-603. [PMID:24501572]

35. Hurley MV, Walsh NE. Effectiveness and clinical applicability of integrated rehabilitation programs for knee osteoarthritis. Curr Opin Rheumatol. 2009;21(2):171-76. [PMID:19339929] http://dx.doi.org/10.1097/BOR.0b013e3283244422

36. 36. Reeuwijk KG, de Rooij M, van Dijk GM, Veenhof C, Steultjens MP, Dekker J. Osteoarthritis of the hip or knee: Which coexisting disorders are disabling? Clin Rheumatol. 2010;29(7):739-47. [PMID:20177725] http://dx.doi.org/10.1007/s10067-010-1392-8

37. Dawson J, Linsell L, Zondervan K, Rose P, Carr A, Randall T, Fitzpatrick R. Impact of persistent hip or knee pain on overall health status in elderly people: A longitudinal population study. Arthritis Rheum. 2005;53(3):368-74. [PMID:15934104] http://dx.doi.org/10.1002/art.21180

38. Hopman-Rock M, Westhoff MH. The effects of a health educational and exercise program for older adults with osteoarthritis for the hip or knee. J Rheumatol. 2000;27(8): 1947-54. [PMID:10955337]

39. Skou ST, Odgaard A, Rasmussen JO, Roos EM. Group education and exercise is feasible in knee and hip osteoarthritis. Dan Med J. 2012;59(12):A4554. [PMID:23290290]

40. Coleman S, Briffa NK, Carroll G, Inderjeeth C, Cook N, McQuade J. A randomised controlled trial of a selfmanagement education program for osteoarthritis of the knee delivered by health care professionals. Arthritis Res Ther. 2012;14(1):R21. [PMID:22284848]

http://dx.doi.org/10.1186/ar3703

41. Heuts PH, de Bie R, Drietelaar M, Aretz K, Hopman-Rock $\mathrm{M}$, Bastiaenen $\mathrm{CH}$, Metsemakers JF, van Weel $\mathrm{C}$, van Schayck O. Self-management in osteoarthritis of hip or knee: A randomized clinical trial in a primary healthcare setting. J Rheumatol. 2005;32(3):543-49.

[PMID:15742451]

42. Simsek H, Doganay S, Budak R, Ucku R. Relationship of socioeconomic status with health behaviors and self-perceived health in the elderly: A community-based study, Turkey. Geriatr Gerontol Int. 2014;14(4):960-68.

[PMID:24118995]

http://dx.doi.org/10.1111/ggi.12166

43. Kaleta D, Polańska K, Dziankowska-Zaborszczyk E, Hanke W, Drygas W. Factors influencing self-perception of health status. Cent Eur J Public Health. 2009;17(3):122-27. [PMID:20020600]

44. Carvalho NA, Bittar ST, Pinto FR, Ferreira M, Sitta RR. Manual for guided home exercises for osteoarthritis of the knee. Clinics (Sao Paulo). 2010;65(8):775-80. [PMID:20835554] http://dx.doi.org/10.1590/S1807-59322010000800006

45. Hochberg MC, Altman RD, April KT, Benkhalti M, Guyatt G, McGowan J, Towheed T, Welch V, Wells G, Tugwell P; American College of Rheumatology. American College of Rheumatology 2012 recommendations for the use of nonpharmacologic and pharmacologic therapies in osteoarthritis of the hand, hip, and knee. Arthritis Care Res (Hoboken). 2012;64(4):465-74. [PMID:22563589] http://dx.doi.org/10.1002/acr.21596

46. Jevsevar DS, Brown GA, Jones DL, Matzkin EG, Manner PA, Mooar P, Schousboe JT, Stovitz S, Sanders JO, Bozic KJ, Goldberg MJ, Martin WR 3rd, Cummins DS, Donnelly P, Woznica A, Gross L; American Academy of Orthopaedic Surgeons. The American Academy of Orthopaedic Surgeons evidence-based guideline on: treatment of osteoarthritis of the knee, 2nd edition. J Bone Joint Surg Am. 2013;95(20):1885-86. [PMID:24288804]

47. Pisters MF, Veenhof C, Schellevis FG, De Bakker DH, Dekker J. Long-term effectiveness of exercise therapy in patients with osteoarthritis of the hip or knee: A randomized controlled trial comparing two different physical therapy interventions. Osteoarthritis Cartilage. 2010;18(8): 1019-26. [PMID:20488250]

http://dx.doi.org/10.1016/j.joca.2010.05.008

48. Braunholtz DA, Edwards SJ, Lilford RJ. Are randomized clinical trials good for us (in the short term)? Evidence for a "trial effect". J Clin Epidemiol. 2001;54(3):217-24. [PMID:11223318] http://dx.doi.org/10.1016/S0895-4356(00)00305-X 
49. Osborne RH, Buchbinder R, Ackerman IN. Can a diseasespecific education program augment self-management skills and improve Health-Related Quality of Life in people with hip or knee osteoarthritis? BMC Musculoskelet Disord. 2006;7:90. [PMID:17134516]

http://dx.doi.org/10.1186/1471-2474-7-90

Submitted for publication August 28, 2014. Accepted in revised form February 17, 2015.

This article and any supplementary material should be cited as follows:

da Silva FS, de Melo FE, do Amaral MM, Caldas VV,
Pinheiro ÍL, Abreu BJ, Brito Vieira WH. Efficacy of simple integrated group rehabilitation program for patients with knee osteoarthritis: Single-blind randomized controlled trial. J Rehabil Res Dev. 2015;52(3):309-22. http://dx.doi.org/10.1682/JRRD.2014.08.0199

ResearcherID: Flávio S. da Silva, MS: E-8925-2015; Bento J. Abreu, PhD: E-2607-2015

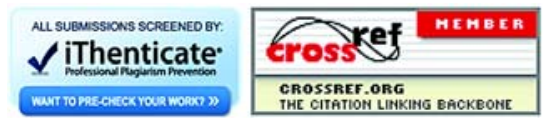


